\title{
El factor extralingüístico en la traducción (francés- español) de expresiones marcadas culturalmente: relación sine qua non en el proceso traslativo
}

\author{
The extralinguistic factor in the translation \\ (French-Spanish) of cultural expressions: a sine \\ qua non relation in the translational process
}

\author{
JoRge VALDEnebro SÁNCHEZ \\ Université de Lorraine \\ jorge.valdenebro-sanchez@univ-lorraine.fr
}

\begin{abstract}
This work addresses the importance of extralinguistic factors in the field of translation. In particular, we focus on the importance of these factors in the of the translations of a series of expressions obtained from the films L'auberge espagnole and Qu'est-ce qu'on a fait au Bon Dieu?, as done by the students in the grado in Traducción e Interpretación of the University of Malaga and by those of the Alliance Française of Malaga. First, we begin with a theoretical course focused on the importance of cultural knowledge for the translator. Secondly, we present the results of the survey made by the students of the two institutions mentioned above in order to be able to compare the results obtained and confirm what we defend in our work: the importance of the extralinguistic factor in translation. Finally, we finish the work with the conclusions, where we will outline the working approach I would like to follow.
\end{abstract}

Key-words

extralinguistic factor, culture, cultural expressions, survey, translation.

\begin{abstract}
Resumé
Ce travail aborde l'importance qu'ont les facteurs extralinguistiques dans le domaine de la traduction. Particulièrement, nous nous focalisons sur l'importance desdits facteurs dans l'analyse des traductions des étudiants du Grado en Traducción e Interpretación de l'Université de Malaga et ceux de l'Alliance Française de Malaga d'une série d'expressions extraites des films L'auberge espagnole et Qu'est-ce qu'on a fait au Bon Dieu? Premièrement, un parcours théorique est établi dans le but de mettre l'accent sur l'importance qu'ont les connaissances culturelles chez le traducteur. Deuxièmement, les résultats de l'enquête qui a été distribuée aux étudiants des deux institutions susmentionnées sont présentés dans le but de pouvoir comparer les résultats obtenus et de confirmer ce que nous défendons dans notre travail: l'importance $\mathrm{du}$ facteur extralinguistique en traduction. Finalement, les conclusions de l'étude, dans lesquelles sont aussi définies les lignes de travail à suivre, sont exposées.
\end{abstract}

\section{Mots-clés}

facteur extralinguistique, culture, expressions culturelles, enquête, traduction. 


\section{Introducción}

En traducción, como bien sabemos, el factor extralingüístico desempeña un papel fundamental. Es cierto que se deben conocer los idiomas con los que se trabaja a un nivel altísimo. Sin embargo, no se puede pasar por alto la importancia de los conocimientos extralingüísticos, pues estos últimos son de vital importancia en las fases de comprensión y reexpresión (Seleskovitch, 1968). Sin ellos, el traductor o intérprete que solo cuente con buenos conocimientos lingüísticos podría verse en la situación de encontrarse con un texto o discurso que le resultase incomprensible, lo que daría lugar a cometer errores en el proceso traslativo como, por ejemplo, el falso sentido. La importancia del factor extralingüístico queda reflejada en la propuesta de PACTE (2003) al considerar la competencia extralingüística como una de las subcompetencias que comprende la competencia traductora (competencia que diferencia al traductor-intérprete del "mero" conocedor de lenguas, cuyo objetivo es la simple comunicación). Por ese motivo, la idea de este trabajo surge de la importancia que tienen los conocimientos culturales en traducción. Este punto es fundamental, ya que consideramos que existe la falsa idea, por parte de todas aquellas personas ajenas a este campo, así como por parte de traductores en formación (sobre todo aquellos que se encuentran en los primeros años de estudio), de que en traducción los conocimientos lingüísticos cuentan con una relevancia mayor que los aspectos extralingüísticos. Para reafirmar nuestro razonamiento, llevamos a cabo un recorrido teórico que nos permite sustentar nuestro estudio práctico de 2017, que consistió en la realización de unas encuestas en ámbitos en los que se trabaja con la lengua francesa, pero bajo diferentes perspectivas: la Alianza Francesa de Málaga y la Universidad de Málaga (estudiantes del grado de Traducción e Interpretación). En dichas encuestas (totalmente anónimas) pedimos que se nos diese una propuesta de traducción de doce expresiones (contextualizadas visualmente) de las películas L'auberge espagnole y Qu'est-ce qu'on a fait au Bon Dieu? con el fin de valorar los resultados y comprobar si se había cumplido nuestra idea de partida: la importancia de lo extralingüístico en traducción.

\section{Objetivos y metodología}

Con la realización de este trabajo pretendemos alcanzar un objetivo principal: corroborar todo lo que diferentes estudios (Nida \& Taber, 1969; Margot, 1979; Reiss \& Vermeer, 1984; Witte, 1987; Snell-Hornby, 1988; etc.) han demostrado acerca de la importancia que tienen los factores extralingüísticos en traducción. Para lograr este objetivo principal, nos hemos tenido que fijar, asimismo, los siguientes objetivos secundarios:

- Analizar las películas mencionadas en el apartado 1 de este trabajo para poder extraer aquellas expresiones que tenían un contenido cultural importante de cara a la traducción. 
- Realizar una encuesta en la que se pide, tanto a alumnos del Grado en Traducción e Interpretación de la Universidad de Málaga como a alumnos de la Alianza Francesa de Málaga, una propuesta de traducción de las doce expresiones finales que han sido objeto de estudio. Con ello se pretende analizar los resultados obtenidos y reafirmar la importancia que tiene el aspecto extralingüístico en traducción e interpretación.

Para poder lograr estos objetivos, hemos seguido la metodología que a continuación se describe, que consta de las fases que pasamos a enumerar y explicar muy brevemente, a continuación. En primer lugar, realizamos un análisis de las dos películas con el objetivo de extraer una lista de expresiones con las que trabajar. La razón por la cual escogimos estas dos películas se debe al alto contenido cultural que en ellas hay. Debido al gran número de expresiones extraídas (en torno a unas trescientas), redujimos la lista a doce teniendo en cuenta el tiempo con el que contábamos en el aula para realizar la encuesta (unos 20 minutos), así como el nivel lingüístico de los alumnos para poder traducir las expresiones sin ningún tipo de ayuda documental (ya que lo que pretendíamos ver en el estudio no era la subcompetencia instrumental con la que contaban, sino la extralingüística exclusivamente).

El siguiente paso lo conforman la clasificación y el análisis de las expresiones. Esta etapa se centró en clasificar las expresiones en una lista categórica creada para tal fin (argot, frase hecha, animales, contenido histórico, contenido jurídico, educación, bebida, léxicas o gramaticales, religión, verlan, ironía, ropa, colores, comida, topónimos, antropónimos, gentilicio, partes del cuerpo, referencia a personajes famosos, películas, marcas, etc.) ${ }^{1} \mathrm{y}$, por último, analizar el problema de traducción de las expresiones, proponer una o varias traducciones a la ya propuesta en la traducción oficial de las películas, comentar aspectos interesantes para el proceso traslativo (dificultades, origen de la expresión, aspectos que debemos tener en cuenta, etc.).

A continuación, pasamos a la elaboración de la encuesta que se realizó en los sectores antes mencionados. Mediante esta encuesta se solicitó a alumnos de diferentes entidades que trabajan con la lengua francesa, mencionados en el apartado anterior, una propuesta de traducción de las expresiones que han sido objeto de estudio. De este modo, pudimos ver los resultados obtenidos de las encuestas realizadas por parte de los alumnos que estudian la lengua francesa desde diferentes puntos de vista, comentar los errores encontrados con mayor frecuencia, analizar por qué se habían producido y verificar así la importancia que tienen los factores extralingüísticos en traducción.

Una vez terminado este paso, acabamos redactando las conclusiones oportunas y las líneas futuras de investigación que nos marcamos con este trabajo.

1 No obstante, esta parte del trabajo no consta en este artículo, ya que forma parte de otro estudio con vistas a una posible publicación. 


\section{La importancia del factor extralingüístico en traducción}

La traducción es una actividad que se da entre dos culturas, no exclusivamente entre dos lenguas. Nosotros entendemos que lengua y cultura no pueden ir por separado, ya que constituyen un binomio complementario y fundamental en el proceso traslativo. Este hecho ya comienza a ponerse de relieve por traductólogos con el giro cultural de finales de los años 80 (Bassnett \& Lefevere, 1990). Autores como Nida \& Taber (1969), Margot (1979), Reiss \& Vermeer (1984), Witte (1987) y Snell-Hornby (1988), entre otros, consideran la traducción como comunicación intercultural y ponen de relieve la importancia de los elementos culturales en traducción. Todas las teorías defendidas por los autores anteriormente mencionados nos hacen ver que aquellos aspectos que no son exclusivamente lingüísticos son de vital importancia en el proceso de traducción. De hecho, Margot afirma que "al esfuerzo de comprensión del texto original, situándolo en su contexto en un sentido amplio (literario, histórico, geográfico, cultural), el traductor debe efectuar un estudio, a la vez lingüístico y cultural, del medio al que se destina la traducción" (Margot, 1979: 36); esto es, a la cultura meta. Asimismo, la importancia de los elementos culturales queda presente en la clasificación del modelo holístico de PACTE (2003), donde el factor extralingüístico tiene un papel clave en la competencia traductora. Según este modelo, la competencia traductora, es decir, "el sistema subyacente de conocimientos, habilidades y actitudes necesarios para traducir" (PACTE, 2001: 39) se compone de la subcompetencia bilingüe, la subcompetencia extralingüística, la subcompetencia de conocimientos sobre la traducción, la subcompetencia instrumental, la subcompetencia estratégica y los componentes psicofisiológicos.

Como vemos, este modelo establece que el factor extralingüístico, es decir, aquellos conocimientos sobre el mundo en general y sobre aspectos particulares de este, es una de las subcompetencias que conforma la competencia traductora. Por lo tanto, estamos ante un aspecto que es tan importante como el factor lingüístico. La falta de conocimientos extralingüísticos, como veremos en nuestro estudio, da lugar a graves errores en la traducción, como el falso sentido o el sin sentido. Por ello, el traspaso de ese aspecto extralingüístico de una lengua a otra supone uno de los principales retos a los que el traductor debe hacer frente y que explicaremos en el siguiente subapartado.

\subsection{Retos en la traducción de expresiones marcadas culturalmente}

Si bien es cierto que el conocimiento de los factores extralingüísticos en traducción resulta crucial en la labor traslativa, estos suponen uno de los principales problemas a los que el traductor debe hacer frente, tal y como afirma Hurtado: "el trasvase de los elementos culturales presentes en un texto es uno de los mayores problemas a que se enfrenta el traductor" (Hurtado, 2011: 607). Ese trasvase cultural en traducción supone, como vemos, un reto tan 
importante que autores como Catford (1970) consideran que es imposible traducir ese factor extralingüístico, produciéndose lo que él denomina intraducibilidad cultural. En cualquier caso, más allá de afirmar lo que las diferentes teorías sostienen acerca de la imposibilidad de traducir los elementos culturales, no podemos negar que la traducción de los culturemas (Vermeer, 1983), entendidos como aquellas unidades semióticas con ideas que contienen un contenido cultural presentes en un texto (Luque Durán, 2009) resulta un reto importante en el proceso traslativo. Por ese motivo, el traductor o intérprete es considerado no simplemente como una figura lingüística, sino como un mediador entre culturas (Hatim \& Mason, 1997). Esto implica que, para la comprensión de un texto que contenga un alto contenido de culturemas, es imprescindible el conocimiento de la cultura de origen (o, en su defecto, paliar las carencias existentes con una buena documentación) y, además, tener un gran conocimiento de la cultura de destino de la lengua a la que se va a traducir. Por consiguiente, cuando nos enfrentamos a una traducción debemos, antes de nada, reflexionar acerca de los posibles problemas culturales que puedan existir entre las diferentes culturas con las que estamos trabajando. Existe un gran número de autores que ofrecen propuestas en este sentido. Nida (1945) propone lo siguiente:

- Diferencia de ecología

- Diferencia de cultura material

- Diferencia de cultura social

- Diferencia de cultura religiosa

- Diferencia de cultura lingüística

Por su parte, Vlakhov \& Florin (1970) crearon el término realia, que hace referencia a aquellos elementos textuales que "denotan color histórico local" (Hurtado, 2011: 608). Dichos elementos textuales son catalogados en cuatro tipos:

- Geográficos y etnográficos

- Folclóricos y mitológicos

- Objetos cotidianos

- Elementos sociohistóricos

Igualmente, Newmark (1988/1992: 133-146) propone lo que él denomina palabras culturales extranjeras y sugiere las siguientes categorías:

- Ecología

- Cultura material

- Cultura social

- Organizaciones, costumbres, actividades, procedimientos y conceptos

- Hábitos y gestos 
EL FACTOR EXTRALINGÜÍSTICO EN LA TRADUCCIÓN (FRANCÉS-ESPAÑOL) DE EXPRESIONES..

También Nord (1994) propone que todos aquellos elementos que no son iguales en las culturas que se van a abordar suponen un punto rico. Estos puntos ricos forman la barrera cultural entre las comunidades en cuestión. Además, Nord lo relaciona con las funciones del lenguaje:

- Función fáctica: es aquella que se encarga de "cómo se desarrolla la comunicación entre el emisor y el receptor" (Nord, 2010: 244). Dicha función tiene cuatro subfunciones: apertura del contacto, mantenimiento del contacto, cierre del contacto, y desarrollo de la relación.

- Función referencial: las diferencias se manifiestan en relación con la representación de objetos y fenómenos y en la forma en que se informa en el texto de dichos fenómenos.

- Función expresiva: esta función hace referencia al emisor, "que puede expresar su actitud frente a las cosas y los fenómenos del mundo, evaluándolos, o sus emociones, sentimientos positivos o negativos, etc." (Nord, 2010: 245). Tiene diferentes subfunciones como, por ejemplo, la emotiva (expresión de emociones), la evaluadora o la ironía.

Finalmente, Katan (1999: 45 y ss.) plantea varios niveles lógicos para poder organizar la información cultural de una manera jerárquica:

- El entorno: constituido por diferentes factores como, por ejemplo, el físico, el clima, la comida, la forma de vestir, etc., y que se van a expresar de forma diferente en cada cultura.

- La conducta: reglas y restricciones de comportamiento de cada cultura.

- Las capacidades, estrategias y habilidades para comunicarse: hace referencia a la manera de transmitir y percibir los mensajes.

- Los valores: aquellos valores que tiene la misma sociedad y jerarquía.

- Las creencias: motivaciones y razones que se dan para seguir reglas de conducta y hacer o no ciertas cosas.

- La identidad: se trata del nivel superior de jerarquía, por lo que es la que conforma y domina el resto de niveles.

Por lo tanto, vemos que existen diferentes propuestas al respecto. La dificultad y la importancia que tienen los diferentes elementos que conforman cada cultura es una realidad a la que todo traductor debe hacer frente. Debido al gran problema que la transferencia de los elementos culturales supone para el traductor e intérprete, existen autores, como Newmark (1988/1992), que señalan procedimientos técnicos de traducción que serían de utilidad para poder llevar a cabo la transposición cultural mencionada anteriormente. Este autor propone el préstamo, el equivalente cultural, la neutralización (es decir, explicación del referente 
cultural), la traducción literal, la naturalización (es decir, la adaptación a la pronunciación, ortografía y morfología de la lengua de llegada), el análisis componencial o explicitación de los rasgos del referente cultural, la supresión, el doblete (es decir, combinación de varias técnicas), la traducción estándar aceptada, la paráfrasis, las glosas y las notas como procedimientos técnicos de traducción.

A la vista de estas propuestas, sería oportuno tener en cuenta varios aspectos. Debemos considerar la relación que existe entre las dos culturas y, además, puede darse el caso de que exista una inequivalencia cultural, falsos amigos culturales, etc. Igualmente, no se puede pasar por alto el género textual. El culturema puede aparecer en cualquier ámbito y en cualquier género textual, por lo que según los casos en los que nos encontremos, las soluciones variarán. Además, hemos de tener en cuenta también la función del culturema. En otras palabras, la relevancia que este tiene dentro del texto. Asimismo, otro punto muy importante sería la naturaleza del culturema (registro, universalidad, etc.), así como las características del destinatario (nivel cultural o motivación). Por último, y no por ello menos importante, la finalidad de la traducción, según la cual el traductor deberá optar por una traducción u otra.

\section{Análisis de los resultados}

Como hemos explicado anteriormente, una de las partes de este estudio la compone la realización de una encuesta en diferentes sectores relacionados con la enseñanza de la lengua francesa. Se trata de una encuesta anónima en la que no solo se pedía la traducción de las expresiones que han sido objeto de análisis en el presente trabajo, sino también ciertos datos de los encuestados relevantes para el estudio como pueden ser la nacionalidad, el tiempo que llevan estudiando francés, la edad, etc.

La encuesta estaba acompañada de un vídeo que elaboramos en el que aparecen las secuencias de las películas donde se dicen las expresiones que son objeto de estudio para poder situar a los encuestados en un contexto óptimo. Una vez realizada la encuesta, procedimos a analizar los resultados obtenidos. En primer lugar, veremos, de forma general, los datos obtenidos: porcentajes de personas que han traducido correctamente las expresiones, de qué institución procedían, errores encontrados en cada una de las expresiones, etc. y, en segundo lugar, haremos una breve reflexión sobre ellos.

Si analizamos cuántas expresiones han contestado bien, de manera global, nuestros encuestados, comprobamos que de los 125 encuestados solo un $0,80 \%$ ha contestado todas las expresiones (12) correctamente; el 2,40\%, entre 10 y 12; el 29,60\%, entre 7 y 9; el $43,20 \%$, entre 4 y 6 ; y el $24 \%$, menos de cuatro. Es un dato curioso, porque hay un porcentaje muy alto de pocas expresiones traducidas correctamente. El siguiente gráfico ilustra estos resultados: 


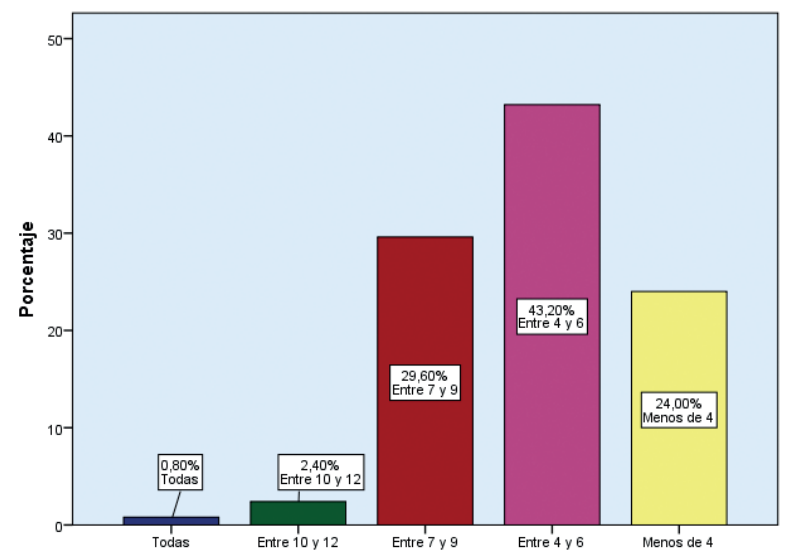

Tabla 1: ¿Cuántas expresiones se ha traducido correctamente?

Resulta aún más interesante ver de qué instituciones provienen las traducciones correctas. Del total de los 125 encuestados, 107 proceden de la Universidad de Málaga, del Grado en Traducción e Interpretación y 18 de la Alianza Francesa de Málaga. Hemos de justificar el reducido número de esta última institución, pues se trata de un centro que no imparte clases a más de 6-8 alumnos por clase y donde los grupos de niveles más avanzados de B2 y C1 suelen ser más escasos. Aun así, los resultados son bastante útiles y no nos basamos en el número de alumnos, sino en el porcentaje en sí. La comparación entre la institución de procedencia y cuántas expresiones se han traducido correctamente es la siguiente:

- Todas las expresiones correctamente traducidas:

o $\quad 0,80 \%$, de la Universidad de Málaga.

- $\quad$ Entre 10 y 12 expresiones correctamente traducidas:

o Alianza Francesa de Málaga: 5,6 \%

o Universidad de Málaga: 1,9\%

- $\quad$ Entre 7 y 9 expresiones correctamente traducidas:

o Alianza Francesa de Málaga: 44,4 \%

o Universidad de Málaga: 27,1 \%

- $\quad$ Entre 4 y 6 expresiones correctamente traducidas:

o Alianza Francesa de Málaga: 38,9 \%

o Universidad de Málaga: 43,9\%

- Menos de 4 expresiones correctamente traducidas:

o Alianza Francesa de Málaga: 11,1\%

o Universidad de Málaga: 26,2 \%

Presentamos aquí la tabla con estos resultados: 


\begin{tabular}{|c|c|c|c|c|c|c|}
\hline \multirow{2}{*}{ Institución } & \multicolumn{5}{|c|}{ ¿Cuántas expresiones ha traducido correctamente? } & \multirow{2}{*}{ Total } \\
\hline & Todas & Entre 10 y 12 & Entre 7 y 9 & Entre 4 y 6 & Menos de 4 & \\
\hline \multirow{2}{*}{$\begin{array}{l}\text { Alianza Francesa } \\
\text { de Málaga }\end{array}$} & 0 & 1 & 8 & 7 & 2 & 18 \\
\hline & $0,0 \%$ & $5,6 \%$ & $44,4 \%$ & $38,9 \%$ & $11,1 \%$ & $100,0 \%$ \\
\hline \multirow{2}{*}{$\begin{array}{l}\text { Universidad de } \\
\text { Málaga. Grado } \\
\text { en Traducción e } \\
\text { Interpretación }\end{array}$} & 1 & 2 & 29 & 47 & 28 & 107 \\
\hline & $0,9 \%$ & $1,9 \%$ & $27,1 \%$ & $43,9 \%$ & $26,2 \%$ & $100,0 \%$ \\
\hline \multirow{2}{*}{ Total } & 1 & 3 & 37 & 54 & 30 & 125 \\
\hline & $0,8 \%$ & $2,4 \%$ & $29,6 \%$ & $43,2 \%$ & $24,0 \%$ & $100,0 \%$ \\
\hline
\end{tabular}

Tabla 2: relación entre las expresiones traducidas correctamente y la institución de procedencia.

Para analizar los resultados de esta comparación debemos tener en cuenta que, aunque el número de alumnos pertenecientes a la Alianza Francesa sea menor, porcentualmente la tasa de mejores resultados es mayor que la de los alumnos de la Universidad de Málaga. Pero, ¿por qué los alumnos de Traducción e Interpretación han podido tener peores resultados? La razón, a nuestro modo de ver, es muy sencilla. La formación de estos últimos es mucho más interdisciplinar, por lo que dentro del aula no se pueden centrar tanto en el plano extralingüístico como los alumnos de la Alianza Francesa. Sin embargo, los alumnos de la Alianza Francesa, aunque su formación no se centre en la competencia traductora, están más en contacto con la cultura francófona y, sobre todo, la francesa. Además, el perfil de los estudiantes de ambas instituciones es muy distinto. En el Grado en Traducción e Interpretación hablamos de estudiantes que acceden a la formación sin, a priori, un nivel de partida exigido en las lenguas con las que se trabaja, y están más centrados en formarse como traductores e intérpretes que en el aprendizaje del idioma en sí (aunque este sea fundamental para llegar a tal fin). En cambio, los estudiantes de la Alianza Francesa de Málaga se fijan como objetivo la adquisición de conocimientos lingüísticos y culturales sin tener en cuenta el aspecto traductológico. Suelen ser, además, alumnos con una gran motivación y capacidad de estudio. Por este motivo, el hecho de traducir expresiones culturales exclusivamente les ha valido a los encuestados de la Alianza Francesa para superar a los estudiantes de la Universidad de Málaga. Quizás si hubiésemos pretendido que nos tradujeran un texto completo, el resultado cambiaría, ya que los alumnos de la Alianza no cuentan con la formación traductora adecuada.

Asimismo, para corroborar esto, analizamos los resultados comparando las traducciones de las expresiones con el hecho de haber estado o vivido en algún país francófono. Podemos comprobar que el haber vivido o disfrutado de algún tipo de estancia en algún país 
EL FACTOR EXTRALINGÜÍSTICO EN LA TRADUCCIÓN (FRANCÉS-ESPAÑOL) DE EXPRESIONES...

de habla francesa influye, pues la tasa de respuestas correctas es mucho mayor entre los encuestados que han estado o vivido en algún país cuya lengua vehicular sea el francés.

\begin{tabular}{|c|c|c|c|c|c|c|}
\hline \multirow{2}{*}{$\begin{array}{l}\text { ¿Ha vivido o estado } \\
\text { usted en Francia o } \\
\text { en algún otro país } \\
\text { francófono? }\end{array}$} & \multicolumn{5}{|c|}{ ¿Cuántas expresiones ha traducido correctamente? } & \multirow{2}{*}{ Total } \\
\hline & Todas & Entre 10 y 12 & Entre 7 y 9 & Entre 4 y 6 & Menos de 4 & \\
\hline \multirow{2}{*}{ Sí } & 1 & 2 & 31 & 33 & 20 & 18 \\
\hline & $1,1 \%$ & $2,3 \%$ & $35,6 \%$ & $37,9 \%$ & $23,0 \%$ & $100,0 \%$ \\
\hline \multirow{2}{*}{ No } & 0 & 1 & 6 & 21 & 10 & 107 \\
\hline & $0,0 \%$ & $2,4 \%$ & $15,8 \%$ & $55,3 \%$ & $26,3 \%$ & $100,0 \%$ \\
\hline \multirow{2}{*}{ Total } & 1 & 3 & 37 & 54 & 30 & 125 \\
\hline & $0,8 \%$ & $2,4 \%$ & $29,6 \%$ & $43,2 \%$ & $24,0 \%$ & $100,0 \%$ \\
\hline
\end{tabular}

Tabla 3: relación entre las expresiones traducidas correctamente y haber vivido o estado en un país francófono.

Con estos resultados, comprobamos que las personas que han estado o vivido en un país francófono han tenido mejores resultados. Debemos matizar que en el apartado de personas que han vivido o estado en Francia se incluyen aquellas que han estados de vacaciones, por lo que se puede justificar que en ocasiones la diferencia no sea tan abismal. Por ese motivo, vamos a analizar los resultados de aquellas personas que han estado o vivido en un país francófono teniendo en cuenta el tipo de estancia realizada.

\begin{tabular}{|c|c|c|c|c|c|c|}
\hline \multirow{2}{*}{$\begin{array}{l}\text { Tipo de estancia } \\
\text { realizada }\end{array}$} & \multicolumn{6}{|c|}{ ¿Cuántas expresiones ha traducido correctamente? } \\
\hline & Todas & Entre 10 y 12 & Entre 7 y 9 & Entre 4 y 6 & Menos de 4 & Total \\
\hline \multirow{2}{*}{ Vacaciones } & 0 & 0 & 10 & 21 & 10 & 41 \\
\hline & $0,0 \%$ & $0,0 \%$ & $24,4 \%$ & $51,2 \%$ & $24,4 \%$ & $100,0 \%$ \\
\hline \multirow{2}{*}{ Movilidad Erasmus } & 0 & 1 & 2 & 3 & 1 & 7 \\
\hline & $0,0 \%$ & $14,3 \%$ & $28,6 \%$ & $42,9 \%$ & $14,3 \%$ & $100,0 \%$ \\
\hline \multirow{2}{*}{$\begin{array}{l}\text { Estancias } \\
\text { lingüísticas }\end{array}$} & 0 & 0 & 6 & 5 & 5 & 16 \\
\hline & $0,0 \%$ & $0,0 \%$ & $37,5 \%$ & $31,3 \%$ & $31,3 \%$ & $100,0 \%$ \\
\hline \multirow{2}{*}{ Ha vivido en el país } & 1 & 1 & 10 & 3 & 3 & 18 \\
\hline & $5,6 \%$ & $5,6 \%$ & $55,6 \%$ & $16,7 \%$ & $16,7 \%$ & $100,0 \%$ \\
\hline \multirow{2}{*}{ Total } & 1 & 2 & 28 & 32 & 19 & 82 \\
\hline & $1,2 \%$ & $2,4 \%$ & $34,1 \%$ & $39,0 \%$ & $23,2 \%$ & $100,0 \%$ \\
\hline
\end{tabular}

Tabla 4: relación entre las expresiones traducidas correctamente y el tipo de estancia realizada en un país francófono. 
Como suponíamos, gran parte de quienes habían vivido o estado en el país y habían traducido incorrectamente las expresiones eran personas que solo habían realizado estancias vacacionales, pues, como vemos en esta tabla, los encuestados que habían estado en un país francófono durante más tiempo (ya sea vivir en el país o movilidad Erasmus) o habían realizado estancias lingüísticas tienen un mayor número de expresiones correctamente traducidas.

Además, vamos a ver qué porcentaje de personas que habla francés en el ámbito familiar (teniendo vivas las dos lenguas en su día a día) han realizado correctamente la traducción de las expresiones.

\begin{tabular}{lcccccc}
\hline ¿Habla usted & \multicolumn{5}{c}{ ¿Cuántas expresiones ha traducido correctamente? } \\
\cline { 2 - 7 } $\begin{array}{l}\text { francés dentro del } \\
\text { entorno familiar? }\end{array}$ & Todas & Entre 10 y 12 & Entre 7 y 9 & Entre 4 y 6 & Menos de 4 & Total \\
\hline \multirow{2}{*}{ Sí } & 1 & 1 & 7 & 3 & 1 & 13 \\
\hline \multirow{2}{*}{ No } & $7,7 \%$ & $7,7 \%$ & $53,8 \%$ & $23,1 \%$ & $7,7 \%$ & $100,0 \%$ \\
\hline \multirow{2}{*}{ Total } & $0,0 \%$ & $1,8 \%$ & $27,0 \%$ & $45,9 \%$ & $25,2 \%$ & $100,0 \%$ \\
\hline
\end{tabular}

Tabla 5: relación entre las expresiones traducidas correctamente y el uso de la lengua francesa en entorno familiar

Tal y como vemos en la tabla, el porcentaje de personas que ha tenido mejores resultados se encuentra en aquellas que hablan francés dentro del entorno familiar y que, por lo tanto, se encuentran en contacto constante con las dos culturas. No obstante, el tener nacionalidad francófona o estar diariamente en contacto con los dos idiomas no le es suficiente para tener todas las expresiones traducidas correctamente. Esto corrobora lo expuesto en la parte teórica: para traducir se necesitan una serie de competencias y subcompetencias que diferencian al traductor de los "meros" conocedores de lenguas, pues ser bilingüe no es sinónimo de poder traducir todo en el par de lenguas que se conoce.

A continuación, vamos a proceder a presentar las expresiones que han sido objeto de estudio, su traducción ${ }^{2}$ y la categoría a la que pertenecen según la clasificación que hemos propuesto personalmente y que ya hemos presentado anteriormente ( $c f$. "Objetivos y metodología").

2 Las traducciones que se exponen no son aquellas presentadas de manera oficial en la película, sino las que nosotros proponemos personalmente (aunque alguna de estas pueden coincidir). 
EL FACTOR EXTRALINGÜÍSTICO EN LA TRADUCCIÓN (FRANCÉS-ESPAÑOL) DE EXPRESIONES...

\begin{tabular}{|c|c|c|}
\hline EXPRESIÓN & TRADUCCIÓN & CATEGORÍA \\
\hline Ta gueule & ¡Cierra el pico! & Argot \\
\hline Mais t'es infernal, toi! & ¡Qué pesado eres! & Argot \\
\hline Entre gaulois il faut s'aider & Entre paisanos hay que ayudarse & Contenido histórico \\
\hline Tu m'en veux? & ¿Estás enfadado conmigo? & Léxicas o gramaticales \\
\hline Sabine m'a fait la gueule & Sabine se ha cabreado conmigo & Argot \\
\hline $\begin{array}{c}\text { Si un mec s'intéressait aux } \\
\text { femmes, ce serait le roi du } \\
\text { pétrole }\end{array}$ & $\begin{array}{l}\text { Si un hombre se interesara de } \\
\text { verdad por las mujeres, sería el } \\
\text { rey del mambo }\end{array}$ & Frase hecha \\
\hline Tu vas la fermer? & ¿Te vas a callar? & Léxicas o gramaticales \\
\hline Vous m'ôtez une épine du pied & Me quitáis un peso de encima & Frase hecha \\
\hline $\begin{array}{c}\text { On aurait mieux fait de se } \\
\text { pacser }\end{array}$ & Mejor hacernos pareja de hecho ${ }^{3}$ & Contenido jurídico \\
\hline Ah, la vache! & ¡Joder, hostia! & Argot \\
\hline Je pète un câble & Me pego un tiro & Argot \\
\hline Foutez-moi le camp! & ¡Fuera de mi vista! & Argot \\
\hline
\end{tabular}

Tabla 6: expresiones, traducción y categoría.

En lo concerniente al análisis de los errores ${ }^{4}$ de cada una de las expresiones que han sido objeto de la encuesta, destacamos lo siguiente:

- Ta gueule: menos de la mitad ha realizado una correcta traducción de esta expresión $(44,80 \%)$ y un porcentaje considerable $(9,60 \%)$ no ha sabido responder. Respecto a los errores, podemos señalar los siguientes: falso sentido $(34,40 \%)$, contrasentido $(8 \%)$, sin sentido $(2,40 \%)$ y sobretraducción $(0,80 \%)$. A modo de ilustración, algunas de las traducciones incorrectas que destacamos propuestas por los estudiantes son“itu lengua!", "eres una pesada" y "tranquila", entre otras.

3 Esta traducción sería aceptable en este contexto, ya que el propósito de esta traducción es transmitir el sentido. No podemos obviar que el PACS es un tipo de unión matrimonial característica de Francia y con sus propias connotaciones jurídicas que van a diferenciarlas de la "pareja de hecho" en España. En otros contextos como, por ejemplo, un documento notarial, la traducción que aquí se presenta sería incorrecta.

4 Aunque muchos son los autores que abordan este concepto, para el presente estudio nos hemos centrado en la definición y en la categorización de errores que propone Delisle (1993). 


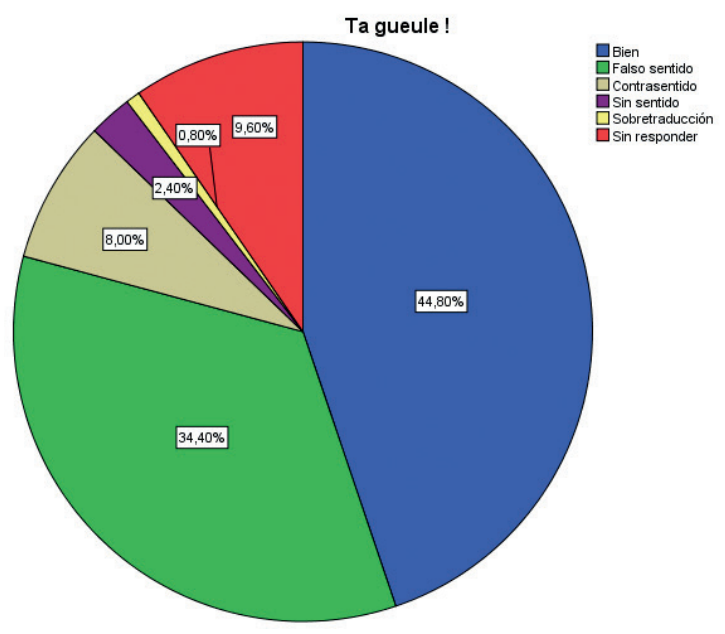

Ilustración 1: ta gueule!

- Mais t'es infernal, toi!: un $52 \%$ ha traducido correctamente esta expresión y un $6,40 \%$ no ha sabido qué responder. Entre los errores más señalados, destacamos el falso sentido (33,60\%), el contrasentido (3,20\%), el sin sentido (0,80\%), la adición $(0,80 \%)$, la hipertraducción (1,60\%) y la sobretraducción (1,60\%). A modo de ilustración, algunas de las traducciones incorrectas que destacamos propuestas por los estudiantes son "ipero qué infernal eres!", "vete al infierno" y "esto es infernal", entre otras.

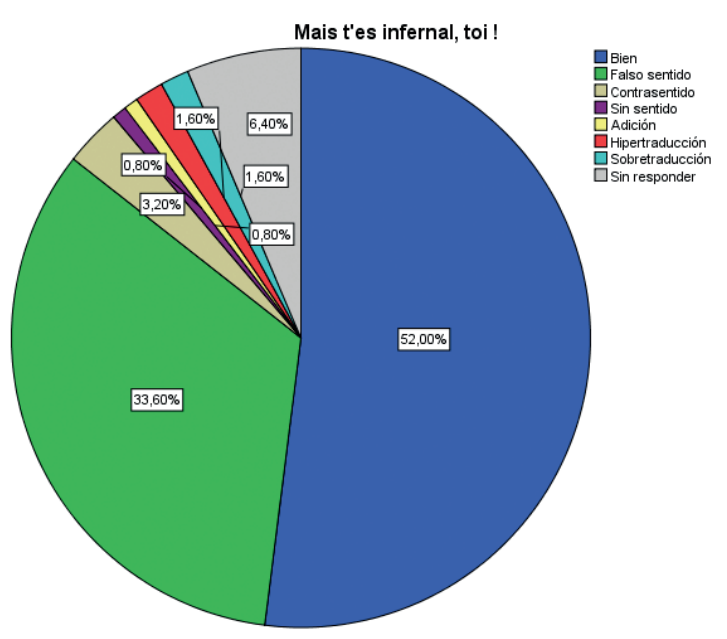

Ilustración 2: mais t’es infernal, toi!

- Entre gaulois il faut s'aider: más de la mitad han traducido correctamente esta expresión $(64 \%)$ frente a aquellos que no han sabido qué poner (2,40\%). Entre los errores come- 
tidos con mayor frecuencia destacamos los siguientes: falso sentido $(28,80 \%)$, hipertraducción (2,40\%), contrasentido (1,60\%) y sobretraducción ( $0,80 \%)$. A modo de ilustración, algunas de las traducciones incorrectas que destacamos propuestas por los estudiantes son "entre todos hay que ayudarse", "los colegas deben ayudarse" y "entre amigos hay que ayudarse", entre otras.

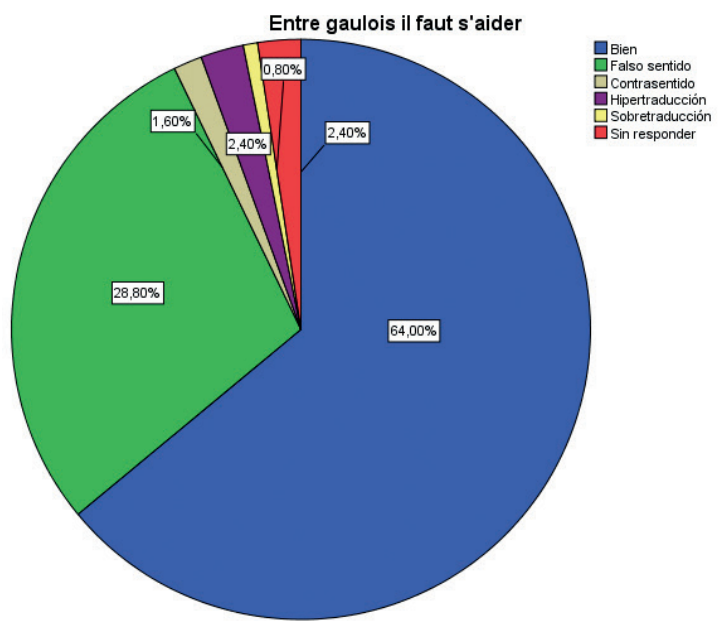

Ilustración 3: entre gaulois il faut s'aider

- Tu m'en veux: esta oración cuenta con una muy baja tasa de éxito $(13,60 \%)$ y una tasa considerable de respuestas en blanco (11,20\%). Entre los errores más frecuentes destacamos el contrasentido (52\%), el falso sentido (16,80\%) y el sin sentido (6,40\%). A modo de ilustración, algunas de las traducciones incorrectas que destacamos propuestas por los estudiantes son “¿me quieres?”, “¿no te importa?” y “ ¿hablas en serio?”, entre otras.

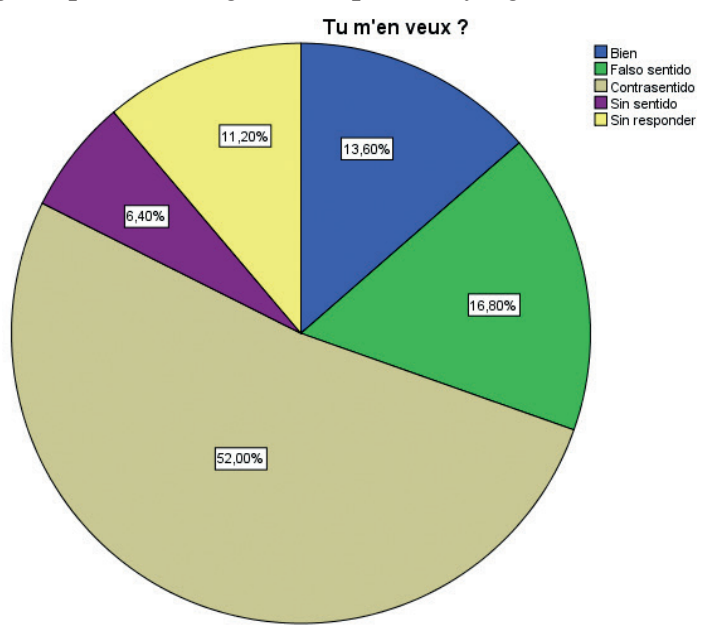

Ilustración 4: tu m’en veux? 
- Sabine m'a fait la gueule: solo un $14 \%$ de los encuestados ha sabido traducir correctamente esta expresión. Un 9,60 \% no ha logrado encontrar la solución y, entre los errores cometidos con mayor frecuencia destacamos los siguientes: falso sentido (44\%), contrasentido $(25,60 \%)$, sin sentido (5,60\%) y sobretraducción (0,80\%). A modo de ilustración, algunas de las traducciones incorrectas que destacamos propuestas por los estudiantes son "Sabine me ha dejado", "Sabine me ha puesto los cuernos" y "Sabine me ha jodido", entre otras.

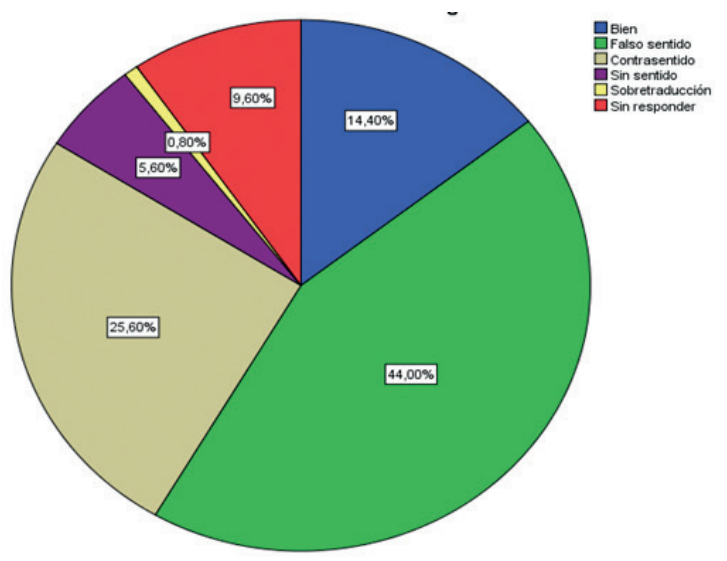

Ilustración 5: Sabine m’a fait la gueule

- Si un mec s'intéressait aux femmes, ce serait le roi du pétrole: un poco menos de la mitad ha traducido correctamente esta expresión (46,40 \%). Como en casos anteriores, hay un porcentaje considerable de personas que no han propuesto ninguna traducción $(14,40 \%)$. En lo que concierne a los errores más frecuentes, destacamos el sin sentido (24\%), la sobretraducción (1,60\%), el falso sentido (5,60\%), el contrasentido $(7,20 \%)$, la hipertraducción $(0,80 \%)$ y la subtraducción (1,60\%). A modo de ilustración, algunas de las traducciones incorrectas que destacamos propuestas por los estudiantes son "si un hombre se interesara en las mujeres, sería la guerra del petróleo", "si un tío se interesara en las mujeres, sería el rey del petróleo" y "si un tío se interesara por las mujeres, sería una suerte", entre otras. 
EL FACTOR EXTRALINGÜÍSTICO EN LA TRADUCCIÓN (FRANCÉS-ESPAÑOL) DE EXPRESIONES...

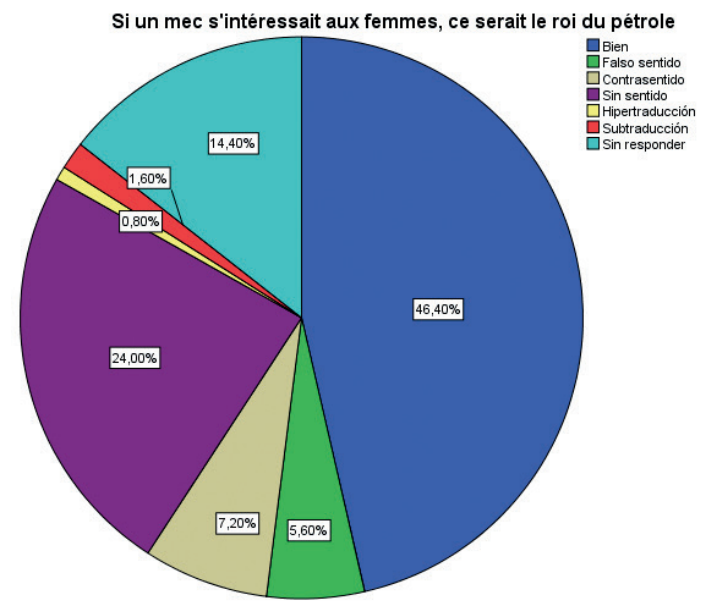

Ilustración 6: si un mec s'intéressait aux femmes, ce serait le roi du pétrole.

- Tu vas la fermer?: los resultados han sido positivos en esta expresión (65,60\% de respuestas correctas). No obstante, podemos destacar las respuestas en blanco (12,80\%) o errores como el falso sentido (14,40\%), la sobretraducción (4\%), el sin sentido (2,40\%) o el contrasentido $(0,80 \%)$. A modo de ilustración, algunas de las traducciones incorrectas que destacamos propuestas por los estudiantes son “¿te la vas a cerrar?” ¿la vas a dejar?” y “ime la vas a quitar?", entre otras.

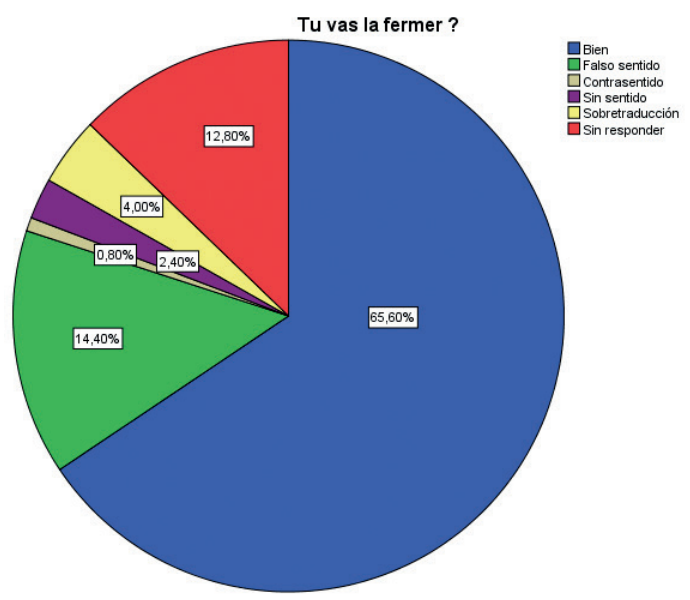

Ilustración 7: tu vas la fermer?

- Vous m'ôtez une épine du pied: menos de la mitad ha realizado la correcta traducción de esta expresión (40,80\%). Además, muchas personas no han, ni siquiera, dado una propuesta de traducción $(20 \%)$. Entre los errores más frecuentes, destacamos los si- 
guientes: contrasentido (15,20\%), falso sentido (11,20\%), sin sentido (8,80\%), subtraducción (3,20\%) y sobretraducción ( $0,80 \%)$. A modo de ilustración, algunas de las traducciones incorrectas que destacamos propuestas por los estudiantes son "me rompéis el corazón", "me quitáis una espina del pie" y "me dais una lección", entre otras.

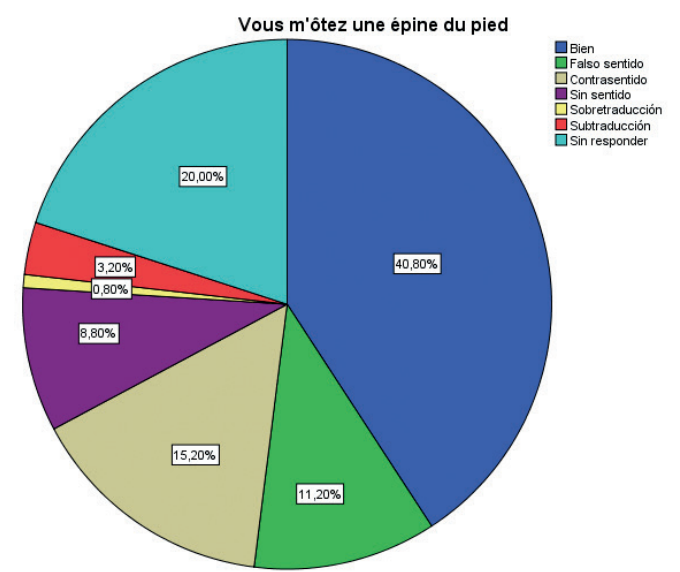

Ilustración 8: vous m’ôtez une épine du pied.

- On aurait mieux fait de se pacser: un porcentaje muy bajo ha logrado traducir correctamente esta expresión $(21,60 \%)$ y uno muy alto se ha quedado sin responder $(22,40 \%)$. Respecto a los errores de traducción, destacamos el contrasentido (18,40\%), el sin sentido $(6,40 \%)$ y la sobretraducción $(0,80 \%)$. A modo de ilustración, algunas de las traducciones incorrectas que destacamos propuestas por los estudiantes son "deberíamos hacer las paces", “sería mejor dejarlo" y "sería mejor que lo dejemos pasar", entre otras.

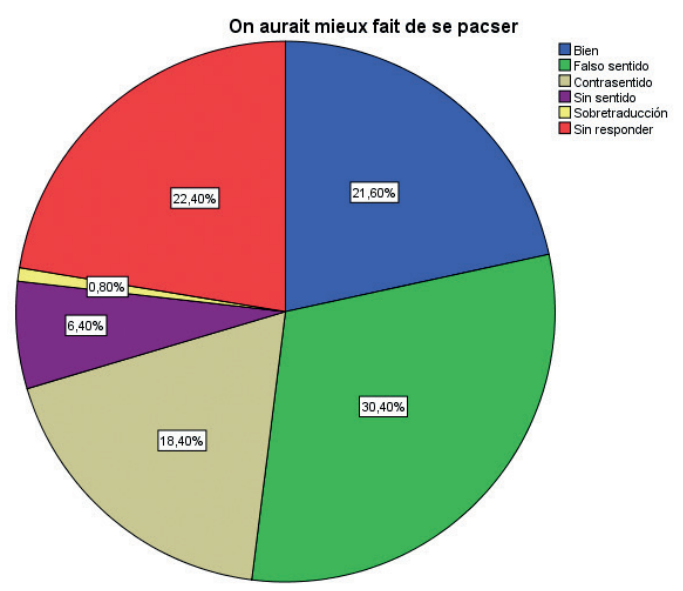

Ilustración 9: on aurait mieux fait de se pacser. 
EL FACTOR EXTRALINGÜÍSTICO EN LA TRADUCCIÓN (FRANCÉS-ESPAÑOL) DE EXPRESIONES...

- Ah, la vache!: un gran porcentaje ha traducido correctamente esta expresión (80 \%). Entre aquellas personas que no se encuentran en este porcentaje destacamos aquellas que no han respondido nada $(11,20 \%)$ y quienes han cometido errores como el falso sentido $(1,60 \%)$, el contrasentido ( $0,80 \%)$, el sin sentido (4\%), la adición $(0,80 \%)$, la sobretraducción $(0,80 \%)$ y la subtraducción $(0,80 \%)$. A modo de ilustración, algunas de las traducciones incorrectas que destacamos propuestas por los estudiantes son “¡ah!”, “ah, iqué duro!”y “¡no veas el bruto!”, entre otras.

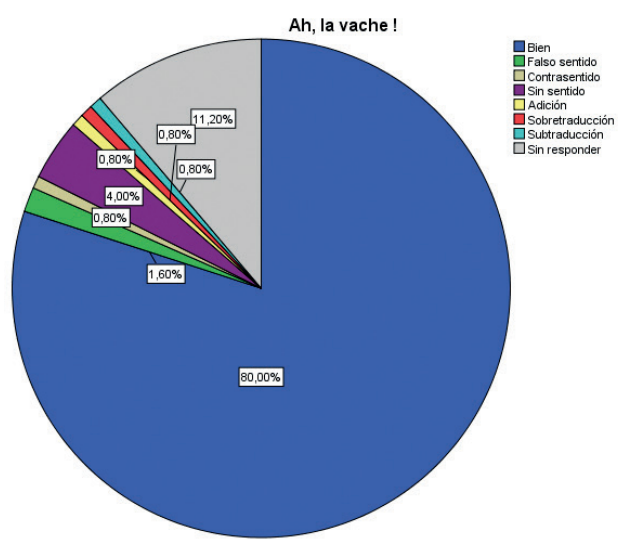

Ilustración 10: ah, la vache!

- Je pète un câble: destacamos el porcentaje elevado de personas sin responder (36\%) y el porcentaje, no demasiado alto, de personas que han dado una traducción correcta a la expresión (32\%). Entre los errores más frecuentes, destacamos los siguientes: falso sentido (20\%), contrasentido $(8,80 \%)$ y el sin sentido (3,20\%). A modo de ilustración, algunas de las traducciones incorrectas que destacamos propuestas por los estudiantes son "yo me tiro un cable", "te echo un cable"y "me quedo muerto", entre otras.

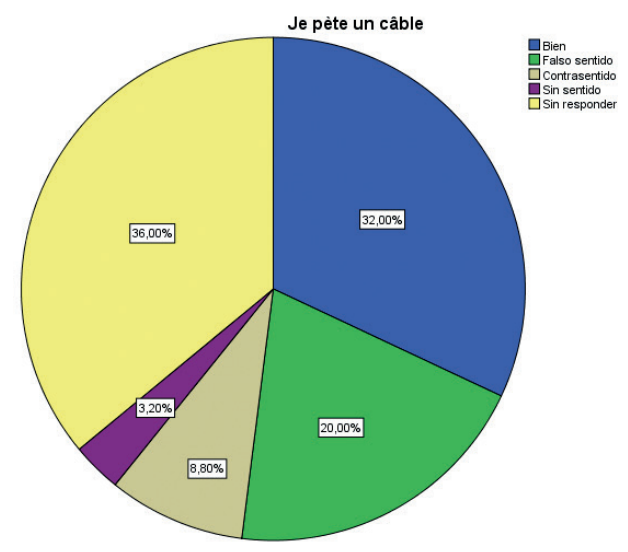

Ilustración 11: je pète un câble. 
- Foutez-moi le camp!: en este caso, hay muchas personas que han traducido correctamente la expresión (73,60 \%). Entre el resto de respuestas, destacamos aquellas que no han propuesto ninguna traducción (10,40\%) o errores como el falso sentido (12\%), el sin sentido $(1,60 \%)$, el contrasentido $(0,80 \%)$, la sobretraducción $(0,80 \%)$ y la subtraducción $(0,80 \%)$. A modo de ilustración, algunas de las traducciones incorrectas que destacamos propuestas por los estudiantes son “¡no me toméis el pelo!”, “no me jodas” y “vayámonos de aquí”, entre otras.

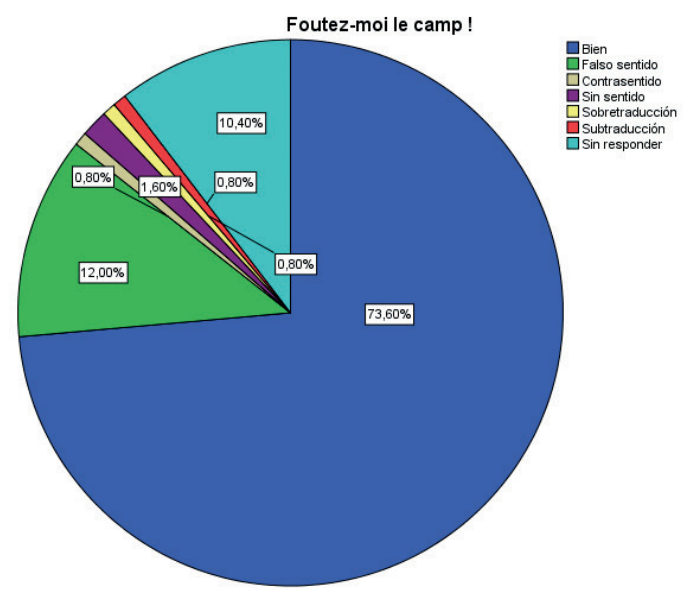

Ilustración 12: foutez-moi le camp!

Una vez que hemos analizado todas las expresiones y tras haber leído los comentarios de los encuestados respecto a la dificultad o dificultades que han encontrado, podemos afirmar varias cosas. En primer lugar, el principal problema que han encontrado para llevar a cabo la traducción de las expresiones se debe a motivos extralingüísticos. Por lo general, comentaron que son expresiones que no se pueden aprender tanto en clase y que traducir palabra por palabra no funciona, pues da lugar a oraciones que no tienen sentido. De hecho, en la mayoría de ocasiones afirmaban conocer las palabras como entidades aisladas (por ejemplo, sabían que gueule era lengua, pero dentro del contexto en cuestión no sabían cómo traducirla). En segundo lugar, podemos comprobar que los errores más frecuentes son el falso sentido y el contrasentido, debido, en gran parte, a la cercanía entre ambos idiomas y ambas culturas). Podemos observar que los traductores e intérpretes en formación encuestados pueden tener un excelente nivel de idiomas, pero se ven superados por los alumnos de la Alianza Francesa en cultura, ya que, en dicha institución, la cultura francófona se estudia con más profundidad (y de manera distinta) que en el Grado en Traducción e Interpretación. Y, en tercer lugar, como consecuencia de todo lo mencionado anteriormente, queda latente que el traductor e intérprete necesita no solo de conocimientos en idiomas, sino de una serie de capacidades (entre ellas, la extralingüística) que los diferencien de los "meros" conocedores de lengua. 


\section{Conclusión}

La realización del presente estudio nos ha llevado a establecer las siguientes conclusiones.

En primer lugar, podemos afirmar lo que comentábamos al principio del presente artículo: el plano extralingüístico es fundamental en traducción. De hecho, comprobamos que la falta de conocimientos extralingüísticos da lugar a errores en la fase de compresión y reexpresión en el proceso traslativo que, a su vez, suponen que el lector de la lengua meta no capte el sentido original del texto. Asimismo, podríamos interpretar, a priori, que los errores de traducción que nos proporcionan nuestras encuestas se deben a una escasa formación extralingüística por parte de nuestras instituciones en cuestión. Aunque, en cierto modo, la formación extralingüística nunca es suficiente, hemos de afirmar que el trabajo de los alumnos fuera de clase para desarrollar las capacidades culturales correspondientes tampoco era el deseado. De hecho, en el apartado de "comentarios" de la encuestas, era el propio alumnado el que afirmaba que trabajaba muy poco o incluso nada la parte cultural del país o países del idioma extranjero que estaba aprendiendo. Por lo general, los encuestados afirmaban que solo se centraban en el trabajo dentro del aula y, a pesar de que se debería incidir más en clase en el plano extralingüístico, también es cierto que con el número de horas con las que se cuenta es imposible llegar a ser un experto en la cultura (en este caso, cultura francófona). Además, en el caso de los alumnos de la Universidad de Málaga los profesores no pueden centrarse tanto en la parte cultural, debido a la disparidad de niveles dentro de un mismo grupo ${ }^{5}$. A nuestro modo de ver, aunque tanto el plano lingüístico como el extralingüístico cuenten con la misma importancia, consideramos que la ayuda del profesional hacia el alumnado es más necesaria en la parte lingüística, ya que la extralingüística puede adquirirse más fácilmente de forma autónoma. Quizás por ese motivo los alumnos de la Alianza Francesa han obtenido mejores resultados 6 . Las clases en esta institución, a diferencia de la Universidad de Málaga, ya están clasificadas por niveles, permitiendo que la progresión sea mucho más rápida y que puedan dedicar más tiempo al plano extralingüístico.

No obstante, sería positivo que los docentes pudiesen llevar a cabo actividades que permitiesen a los alumnos mejorar sus capacidades culturales y lingüísticas al mismo tiempo. Por ejemplo, en nuestro caso, en los estudios de Langues Étrangères Appliquées de la Université de Lorraine, Nancy (France), dentro de la colaboración que hacemos principalmente con la Universidad de Málaga (pero también con otras universidades españolas como la Universidad Pablo de Olavide y la Universidad de Valladolid) en el seno del Proyecto de

5 De hecho, un alumno puede estar matriculado en una asignatura de Lengua y cultura "B" aplicadas a la Traducción e Interpretación (francés) de segundo año sin haber aprobado la asignatura correspondiente de primer año de Grado.

6 Recalcamos que en nuestro estudio nos basamos exclusivamente en la subcompetencia extralingüística en traducción. No tenían ningún tipo de ayuda documental (solo el vídeo que se les puso durante la realización de la encuesta), ya que nuestro objetivo no era ver la capacidad documental. 
Innovación Educativa "Dinamizando: creación de un espacio cultural francófono (ECUF) en la enseñanza-aprendizaje de la lengua y civilización francesas aplicadas a la traducción y la interpretación ${ }^{7 "}$, nuestros alumnos de español realizaron una actividad de intercambio de forma virtual con alumnos de la Universidad de Málaga. A cada alumno francés se le asignó un alumno de Málaga con el fin de que estos, a través de sus idiomas extranjeros correspondientes, establecieran relaciones (en un primer lugar, a través de correo electrónico). Tal actividad permite practicar la expresión escrita y oral de manera real y conocer, a través de la pareja asignada, aspectos de la cultura en cuestión. Asimismo, en el año 2019, realizamos en Nancy un encuentro con estudiantes de la Universidad de Málaga y la Université de Lorraine. Esto permitió a ambos estudiantes mejorar las capacidades lingüísticas de sus idiomas y, en el caso de los estudiantes españoles, conocer la cultura y civilización francesas, estudiadas en clase, de primera mano. La intención de esta última actividad es realizar un futuro encuentro en Málaga para que los alumnos franceses conozcan la cultura española en el propio país. Evidentemente, el intercambio en un país extranjero supone un problema económico, pero vemos que el contacto con universidades de otros países puede permitir la colaboración entre los discentes y realizar un sinfín de actividades culturales de forma, al menos, virtual.

En segundo lugar, podemos afirmar también que el traductor necesita de una serie de competencias que lo diferencie de los "meros" conocedores de lenguas. Ser bilingüe no es sinónimo de saber traducir todo de un idioma a otro. Tal capacidad forma parte de una de las subcompetencias de la competencia traductora (PACTE, 2003). Si bien es cierto que haber vivido en el país o mantener vivas las dos lenguas con las que se trabaja (por el hecho de tener familia de procedencia francófona, por ejemplo) ayuda, vemos que no es suficiente para realizar una traducción correcta de las expresiones. El traductor, como hemos visto, necesita de muchas otras subcompetencias. En los resultados podemos comprobar que existen personas que hablan francés dentro del entorno familiar (por lo que deducimos que tienen la subcompetencia bilingüe bastante desarrollada), pero que esto no les ha valido para traducir la totalidad de expresiones, aunque les haya servido de ayuda. Por ello, los aprendices de idiomas enfocados a la traducción no solo deben centrarse en aspectos gramaticales o conversacionales, que a priori son más complicados, sino trabajar paralelamente las culturas implicadas en el proceso traslativos (además del resto de subcompetencias implicadas en la traducción, ya que cada una de ellas son igual de importantes).

$\mathrm{Y}$, en tercer lugar, la realización de la presente investigación nos anima a realizar un segundo estudio a mayor escala con el objetivo de analizar la situación en España y en Francia en lo concerniente a los conocimientos extralingüísticos en traducción. En el futuro trabajo sería quizás muy interesante llevar a cabo, en primer lugar, el análisis de los problemas y dificultades de un texto con alto contenido cultural por parte de los alumnos con los

7 Se trata de un proyecto cuyo objetivo principal es la creación de un espacio cultural francófono que haga más accesible el proceso de enseñanza (por parte de los docentes) y aprendizaje (por parte de los estudiantes) de la lengua francesa y de las diferentes culturas francófonas del Grado en Traducción e Interpretación. 
que se vaya a trabajar y, posteriormente, realizar dos traducciones: una sin ayuda documental y otra con el uso de fuentes. Con el análisis podríamos ver qué dificultades consideran ellos que tienen (si son más bien de tipo lingüístico, cultural, etc.) y la finalidad de realizar dos traducciones no es más que comparar, en la primera de ellas, el nivel extralingüístico real de los encuestados y, en la segunda, valorar otras subcompetencias, como la instrumental. Esto daría lugar a un trabajo mucho más completo y amplio.

\section{Referencias bibliográficas}

Bassnett-McGuire, Susan \& André Lefevere. 1990. Translation, History and Culture. Londres, Pinter Publishers.

CATFORD, John Cunnison. 1970. Una teoría lingüística de la traducción: ensayo de lingüistica aplicada. Caracas, Universidad Central de Venezuela.

Delisle, Jean. 1993. La Traduction Raisonnée. Manuel d'initiation à la traduction professionnelle de l'anglais vers le français. Ottawa, Les Presses de l'Université d'Ottawa. Colección Pédagogie de la traduction.

Hatim, Basil \& Ian Mason. 1997. The Translator as Communicator. Londres, Routledge.

Hurtado Albir, Amparo. 2011. Traducción y traductología. Introducción a la traductología. Madrid, Ediciones Cátedra.

Katan, David. 1999. Translating Cultures. An Introduction for Translators, Interpreters and Mediators. Ámsterdam, Rodopi.

LuQue Durán, Juan de Dios. 2009. "El diccionario intercultural e interlingüístico y su aplicación a la traducción de culturemas". Ponencia presentada a las III Jornadas Internacionais da Traduçao da Universidade da Evora. Evora, 6 -11 octubre 2009.

LuQue NADAL, Lucía. 2009. “Los culturemas: ¿unidades lingüísticas, ideológicas o culturales?" in Language Design n. . 2, 93-120.

Margot, Jean Claude. 1979. Traduire sans trahir. Lausana, l’Age d'Homme.

Newmark, Peter. 1988. A textbook of Translation. Londres, Prentice Hall (1992. Manual de Traducción. Madrid, Catedra, 1992).

NidA, Eugene \& Charles Taber. 1969. The Sociolinguistics of Interlingual Communication. Bruselas, Les Éditions du Hazard.

NIDA, Eugene. 1945. "Linguistics and Ethnology in Translations Problems" in Word n. ${ }^{\circ}$, 194-208.

Nord, Christiane. 1994. "It's a tea-time in Wonderland. Culture-makers in fictional texts" in Pürschel, Heiner (ed.). Intercultural Communication. Duisburg, Leang.

Nord, Christiane. 2010. "Las funciones comunicativas en el proceso de traducción: un modelo cuatrifuncional" in Nucleo n. ${ }^{\circ} 27,239-255$. 
PACte. 2001. “La competencia traductora y su adquisición” in Quaderns n. ${ }^{\circ}$ 6, 39-45.

Pacte. 2003. "Building a Translation Competence Model" in Alves, Fabio (ed.) Triangulating Translation: Perspectives in Process Oriented Research. Ámsterdam, John Benjamins, 43-66.

ReIss, Khatarina \& Hans-Josef VermeER. 1984. Grundlegung einer allgemeinen Translationstheorie. Tubinga, Niemeyer (Fundamentos para una teoría funcional de la traducción. Madrid, Akal, 1996).

Seleskovitch, Danica. 1968. L'interprète dans les conférences internationales. Problèmes de langage et de communication. Paris, Minard.

Snell-Hornby, Mary. 1988. Translation Studies. An Integrated Approach. Ámsterdam, John Benjamins.

Vermeer, Hans-Josef. 1983. "Translation theory and linguistics” in Roinila, P. Orfanos R. \& S. Tirkkonen-Condit (eds.). Näkökhtia käänämisen tutkimuksesta (eds.). Joensuu, Univ. of Joensuu, 1-10.

VlakHov, Sergei \& Sider FloRIN. 1970. "Neperevodimoe v perevode: realii" in Masterstvo perevoda, Moscú, Sovetskii pisatel, 432 -456.

Witte, Heidrun. 1987. "Die Kulturkompetenz des Translators” in Textcontext n. ${ }^{\circ}$ 2, 109-136. 
